\title{
The Joint Distribution of the Surplus \\ Prior to Ruin and the Deficit at Ruin in Some Sparre Andersen Models
}

by

David C M Dickson

The University of Melbourne

and

Steve Drekic

University of Waterloo

RESEARCH PAPER NUMBER 108

AUGUST 2003

Centre for Actuarial Studies

Department of Economics

The University of Melbourne

Victoria 3010

Australia 


\title{
The joint distribution of the surplus prior to ruin and the deficit at ruin in some Sparre Andersen models
}

\author{
David C M Dickson and Steve Drekic
}

\begin{abstract}
In the Sparre Andersen risk model, we derive a general expression for $h(u, x, y)$, the joint density function of the surplus prior to ruin and the deficit at ruin when the initial surplus is $u$. This density function is expressed in terms of the corresponding density function when the initial surplus is 0 . We apply a known result for $h(0, x, y)$ in the situation when claim inter-arrival times follow a generalised Erlang distribution to derive expressions for $h(u, x, y)$ when individual claims have a phase-type $(m)$ distribution, $m \in Z^{+}$. We also consider the case when claim inter-arrival times follow a phase-type(2) distribution and derive an expression for $h(0, x, y)$. Finally, we illustrate our results with examples.
\end{abstract}

\section{Introduction}

In this paper, we consider the Sparre Andersen risk model. Thus we assume that the time until the first claim and the times between claims form a sequence of independent and identically distributed (i.i.d.) continuous random variables, $\left\{T_{i}\right\}_{i=1}^{\infty}$, with common density function $k$. Let $\left\{X_{i}\right\}_{i=1}^{\infty}$ be a sequence of i.i.d. random variables, where $X_{i}$ denotes the amount of the $i$ th claim. Let $P(x)=1-\bar{P}(x)=\operatorname{Pr}\left(X_{1} \leq x\right), x \geq 0$, denote the distribution function of $X_{1}$. We assume that $P(0)=0$ and that $X_{1}$ is a continuous random variable with density function $p$. We further assume that the sequences $\left\{T_{i}\right\}_{i=1}^{\infty}$ and $\left\{X_{i}\right\}_{i=1}^{\infty}$ are independent. Let $\kappa=E\left(X_{1}\right)$ and $\mu=E\left(T_{1}\right)$.

Let $c$ denote the insurer's premium income per unit time, which we assume to be received continuously. We define the probability of ultimate ruin for 
this risk process by

$$
\psi(u)=\operatorname{Pr}\left(u+\sum_{i=1}^{n}\left(c T_{i}-X_{i}\right)<0 \text { for some } n, n=1,2,3, \ldots\right)
$$

and define $\delta(u)=1-\psi(u)$. It is well known (see, for example, Rolski et al (1999, Section 6.5)) that $\delta(u)=\operatorname{Pr}(L \leq u)$ where the random variable $L$ denotes the maximum of the aggregate loss process. We assume that $c \mu-\kappa>0$, so that $\psi(u)<1$. The implied loading factor is $\theta=c \mu / \kappa-1$.

Let $\{U(t)\}_{t \geq 0}$ denote the surplus process starting from $U(0)=u \geq 0$, and define the time of ruin, $T$, as

$$
T=\left\{\begin{array}{l}
\inf \{t: U(t)<0\} \\
\infty \text { if } U(t) \geq 0 \text { for all } t>0
\end{array}\right.
$$

so that $\psi(u)=\operatorname{Pr}(T<\infty)$. Let $T^{-}$denote the time immediately prior to ruin.

We now define three defective distribution functions. First of all, let

$$
\begin{aligned}
H(u, x, y) & =\int_{0}^{x} \int_{0}^{y} h(u, r, s) d s d r \\
& =\operatorname{Pr}\left(U\left(T^{-}\right) \leq x,|U(T)| \leq y, T<\infty \mid U(0)=u\right)
\end{aligned}
$$

denote the joint distribution function of the surplus prior to ruin and the deficit at ruin, where $h$ denotes the joint density function. Then we can define the marginal distribution functions

$$
F(u, x)=\lim _{y \rightarrow \infty} H(u, x, y) \quad \text { and } \quad G(u, y)=\lim _{x \rightarrow \infty} H(u, x, y)
$$

so that $F$ is the distribution function of the surplus prior to ruin, and $G$ is the distribution function of the deficit at ruin. Let their respective defective density functions be denoted by

$$
f(u, x)=\frac{d}{d x} F(u, x) \quad \text { and } \quad g(u, y)=\frac{d}{d y} G(u, y) .
$$

It is convenient to introduce the proper density function of the deficit at ruin when $u=0$, which we denote $\gamma$, and define by $\gamma(y)=g(0, y) / \psi(0)$. Finally, define the function $\phi$ by

$$
\phi(u)=E\left[w\left(U\left(T^{-}\right),|U(T)|\right) I(T<\infty) \mid U(0)=u\right]
$$

where $w$ is an arbitrary bounded function and $I(A)$ denotes the indicator of an event $A$. 
The remainder of the paper is as follows. In Section 2, we provide a general solution for $H(u, x, y)$, and consequently its joint density function, $h(u, x, y)$. In Section 3, we consider the case in which the density function $k$ has a generalised Erlang distribution (as considered by Gerber and Shiu (2003a, 2003b)) in combination with claim amount density function $p$ being phase-type $(m), m \in Z^{+}$. In Section 4, we consider the case when the density function $k$ is phase-type(2), or, equivalently, Coxian(2). As the phase-type(2) class is not a special case of the generalised Erlang family of distributions, the approach we take in Section 4 is different to that of Section 3 . In fact, our approach leads to a much simpler method of finding $h(u, x, y)$ than that of Cheng and Tang (2003) who consider the situation when $k$ is an Erlang(2) density function, a density function which belongs to both the phase-type(2) and generalised Erlang classes. In Section 5, we give some examples.

\section{A formula for $H(u, x, y)$}

To derive an expression for $H(u, x, y)$, we apply arguments given in Section 6.5 of Rolski et al (1999). We consider first the case when $u \geq x$. In order for the surplus immediately prior to ruin to be less than or equal to $x$, the surplus cannot fall below 0 on the first occasion that it drops below its initial level $u$. Hence it follows that

$$
H(u, x, y)=\int_{0}^{u} g(0, z) H(u-z, x, y) d z=\psi(0) \int_{0}^{u} \gamma(z) H(u-z, x, y) d z .
$$

Taking partial derivatives with respect to $x$ and $y$ yields

$$
h(u, x, y)=\psi(0) \int_{0}^{u} \gamma(z) h(u-z, x, y) d z .
$$

In the case when $0 \leq u<x$, it is possible for ruin to occur at the time the surplus first falls below its initial level $u$, and for the surplus prior to ruin to be less than or equal to $x$, and for the deficit at ruin to be less than or equal to $y$. The probability of this event is

$$
J(u, x, y)=\int_{0}^{x-u} \int_{u}^{u+y} h(0, r, s) d s d r
$$

as the event is equivalent to ruin occurring from initial surplus 0 with a surplus immediately prior to ruin less than or equal to $x-u$ and a deficit at ruin between $u$ and $u+y$. Hence, for $0 \leq u<x$, we have

$$
H(u, x, y)=\psi(0) \int_{0}^{u} \gamma(z) H(u-z, x, y) d z+J(u, x, y)
$$


and

$$
h(u, x, y)=\psi(0) \int_{0}^{u} \gamma(z) h(u-z, x, y) d z+h(0, x-u, u+y) .
$$

Therefore, for $u \geq 0$, we have

$$
H(u, x, y)=\psi(0) \int_{0}^{u} \gamma(z) H(u-z, x, y) d z+Z(u, x, y)
$$

where $Z(u, x, y)=I(u<x) J(u, x, y)$, and

$$
h(u, x, y)=\psi(0) \int_{0}^{u} \gamma(z) h(u-z, x, y) d z+I(u<x) h(0, x-u, u+y) .
$$

Let

$$
H^{*}(s, x, y)=\int_{0}^{\infty} e^{-s u} H(u, x, y) d u
$$

be the Laplace transform of $H$, with similar notation for Laplace transforms of other functions. Then by basic properties of Laplace transforms,

$$
H^{*}(s, x, y)=\frac{Z^{*}(s, x, y)}{1-\psi(0) \gamma^{*}(s)}
$$

From Rolski et al (1999, Section 6.5), $L$ has a compound geometric distribution, and so its Laplace-Stieltjes transform is given by

$$
E\left(e^{-s L}\right)=\int_{0}^{\infty} e^{-s u} d \delta(u)=\frac{\delta(0)}{1-\psi(0) \gamma^{*}(s)}
$$

which implies that

$$
H^{*}(s, x, y)=\frac{E\left(e^{-s L}\right)}{\delta(0)} Z^{*}(s, x, y) .
$$

Since the product of two transforms is the transform of a convolution, it immediately follows that

$$
\begin{aligned}
H(u, x, y) & =\frac{1}{\delta(0)} \int_{0}^{u} Z(u-z, x, y) d \delta(z) \\
& =\frac{1}{\delta(0)} \int_{0}^{u} I(u-z<x) J(u-z, x, y) d \delta(z) .
\end{aligned}
$$


Hence the above equation provides a means of finding $H(u, x, y)$ provided that we know both $\delta$ and $J$. In a similar fashion, we also have the corresponding analogue for $h(u, x, y)$, namely

$$
h(u, x, y)=\frac{1}{\delta(0)} \int_{0}^{u} I(u-z<x) h(0, x-u+z, u-z+y) d \delta(z) .
$$

We remark that equation (6.4) of Dickson and Hipp (2000) for $G(u, y)$ can be obtained from equation (2.2) as

$$
\lim _{x \rightarrow \infty} J(u, x, y)=G(0, u+y)-G(0, u) .
$$

See also Willmot and Lin (1998, p.92). Moreover, Dufresne and Gerber (1988) show that in the classical Poisson risk model

$$
h(0, x, y)=f(0, x) \frac{p(x+y)}{\bar{P}(x)} .
$$

As the arguments underlying this formula equally apply in the Sparre Andersen model, finding $J$ reduces to finding the density function of the surplus prior to ruin when the initial surplus is 0 .

We end this section by remarking that in the classical model $f(0, x)=(\lambda / c) \vec{P}(x)$, so that $h(0, x, y)=(\lambda / c) p(x+y)$ (see, for example, Dufresne and Gerber (1988)). Hence, for $0 \leq u<x$, equation (2.3) yields

$$
h(u, x, y)=\frac{1}{\delta(0)} \int_{0}^{u} \frac{\lambda}{c} p(x+y) d \delta(z)=\frac{\lambda}{c} p(x+y) \frac{1-\psi(u)}{1-\psi(0)},
$$

and for $u>x$ it yields

$$
h(u, x, y)=\frac{1}{\delta(0)} \int_{u-x}^{u} \frac{\lambda}{c} p(x+y) d \delta(z)=\frac{\lambda}{c} p(x+y) \frac{\psi(u-x)-\psi(u)}{1-\psi(0)},
$$

results first given by Dickson (1992).

\section{Generalised Erlang inter-claim times}

In this section we consider the situation where $k$ has a generalised Erlang density function. In particular, we assume that $k$ is the density function of $T_{1}=\sum_{j=1}^{n} W_{j}$ where $\left\{W_{j}\right\}_{j=1}^{n}$ are $n$ independent, exponentially distributed random variables with $E\left(W_{j}\right)=1 / \lambda_{j}$. This generalised Erlang distribution belongs to the phase-type $(n)$ family of distributions and as such, $k$ can be written as

$$
k(t)=\underline{\beta} \exp \{t S\} \underline{S}^{o}, t>0
$$


where $\underline{\beta}=(1,0,0, \ldots, 0,0)$ is a $1 \times n$ row vector,

$$
S=\left[\begin{array}{cccccc}
-\lambda_{1} & \lambda_{1} & 0 & \cdots & 0 & 0 \\
0 & -\lambda_{2} & \lambda_{2} & \cdots & 0 & 0 \\
0 & 0 & -\lambda_{3} & \cdots & 0 & 0 \\
\vdots & \vdots & \vdots & \ddots & \vdots & \vdots \\
0 & 0 & 0 & \cdots & -\lambda_{n-1} & \lambda_{n-1} \\
0 & 0 & 0 & \cdots & 0 & -\lambda_{n}
\end{array}\right]
$$

is an $n \times n$ transition rate matrix, and $\underline{S}^{o}=\left(0,0,0, \ldots, 0, \lambda_{n}\right)^{\prime}$ is an $n \times 1$ column vector. Also, the matrix exponential in equation (3.1) is defined by

$$
\exp \{t S\}=\sum_{j=0}^{\infty} \frac{t^{j}}{j !} S^{j}
$$

For a detailed treatment of phase-type distributions, we refer the reader to Neuts (1981), Latouche and Ramaswami (1999), Rolski et al (1999), and Asmussen (2000).

For this choice of $k$, Gerber and Shiu (2003b) show that

$$
h(0, x, y)=\mathcal{C} p(x+y) \sum_{j=1}^{n} a_{j} e^{-\rho_{j} x}
$$

where $\mathcal{C}=\prod_{r=1}^{n}\left(\lambda_{r} / c\right), a_{j}=\prod_{r=1, r \neq j}^{n}\left(\rho_{r}-\rho_{j}\right)^{-1}$, and $\left\{\rho_{r}\right\}_{r=1}^{n}$ are the $n$ roots in the right half of the complex plane (assumed to be distinct) satisfying the following equation for $\xi$ :

$$
\prod_{j=1}^{n}\left(1-\frac{c}{\lambda_{j}} \xi\right)-p^{*}(\xi)=0 .
$$

We remark that equation (3.3) may be alternatively expressed as $k^{*}(-c \xi) p^{*}(\xi)=1$. Furthermore, it is clear from equation (3.3) that $\xi=0$ is always one of these $n$ roots. Substituting equation (3.2) into equation (2.3), we obtain

$$
h(u, x, y)=\frac{\mathcal{C}}{\delta(0)} p(x+y) \sum_{j=1}^{n} a_{j} e^{-\rho_{j}(x-u)} \int_{0}^{u} I(u-z<x) e^{-\rho_{j} z} d \delta(z) .
$$

Let us now assume that claim amounts have a general phase-type $(m)$ distribution, $m \in Z^{+}$, with density function $p(x)=\underline{\alpha} \exp \{x A\} \underline{A}^{o}$ and corresponding distribution function $P(x)=1-\underline{\alpha} \exp \{x A\} \underline{e}_{m}$ where $\underline{e}_{m}$ denotes 
an $m \times 1$ column vector of ones and $\underline{A}^{o}=-A \underline{e}_{m}$. From Theorem 4.4 in Asmussen (2000), the distribution function of the maximal aggregate loss $L$ in the general Sparre Andersen model with phase-type $(m)$ distributed claim amounts is given by

$$
\delta(u)=1-\underline{\eta} \exp \left\{u\left(A+\underline{A}^{o} \underline{\eta}\right) \underline{e}_{m}, u \geq 0\right.
$$

where the defective row vector $\underline{\eta}$ is the unique solution satisfying the relation

$$
\underline{\eta}=(1 / c) \underline{\alpha} \int_{0}^{\infty} \exp \left\{t\left(A+\underline{A}^{o} \underline{\eta}\right)\right\} k(t / c) d t
$$

However, since $k$ has the phase-type form given by equation (3.1), an explicit solution for $\underline{\eta}$ may actually be obtained. Omitting the tedious mathematical details, it can be verified that

$$
\underline{\eta}=\mathcal{C} \underline{\alpha} \sum_{j=1}^{n} a_{j}\left(\rho_{j} I_{m}-A\right)^{-1}
$$

satisfies equation (3.6) where $I_{m}$ denotes the $m \times m$ identity matrix. Furthermore, it follows from equation (3.5) and the fact that $\underline{A}^{\circ}=-A \underline{e}_{m}$ that the density function of $L$ is given by $\delta^{\prime}(u)=\delta(0) \underline{\eta} \exp \left\{u\left(A+\underline{A}^{\circ} \underline{\eta}\right)\right\} \underline{A}^{\circ}, u>0$, with $\delta(0)=1-\underline{\eta}_{m}$.

Consider first the case when $0 \leq u<x$. In this case, $I(u-z<x)=1$ for all $z \in[0, u]$, and so equation (3.4) becomes

$$
\begin{aligned}
h(u, x, y) & =\frac{\mathcal{C}}{\delta(0)} p(x+y) \sum_{j=1}^{n} a_{j} e^{-\rho_{j}(x-u)}\left[\delta(0)+\int_{0}^{u} e^{-\rho_{j} z} \delta^{\prime}(z) d z\right] \\
& =\mathcal{C} \underline{\alpha} \exp \{(x+y) A\} \underline{A}^{o} \sum_{j=1}^{n} a_{j} e^{-\rho_{j}(x-u)}\left(1+\int_{0}^{u} e^{-\rho_{j} z} \underline{\eta} \exp \{z D\} \underline{A}^{o} d z\right)
\end{aligned}
$$

where we define $D=A+\underline{A}^{o} \underline{\eta}$ for the sake of notational convenience. However, note that

$$
\begin{aligned}
\int_{0}^{u} e^{-\rho_{j} z} \underline{\eta} \exp \{z D\} \underline{A}^{o} d z & =\underline{\eta}\left(\int_{0}^{u} \exp \{z D\} e^{-\rho_{j} z} d z\right) \underline{A}^{o} \\
& =\underline{\eta}\left(\int_{0}^{u} \exp \left\{z\left(D-\rho_{j} I_{m}\right)\right\} d z\right) \underline{A}^{o} \\
& =\underline{\eta} B_{j}^{-1}\left(\exp \left\{u B_{j}\right\}-I_{m}\right) \underline{A}^{o}
\end{aligned}
$$


where we define $B_{j}=D-\rho_{j} I_{m}$. Substituting equation (3.9) into equation (3.8) immediately yields for $0 \leq u<x$

$h(u, x, y)=\mathcal{C} \underline{\alpha} \exp \{(x+y) A\} \underline{A}^{o} \sum_{j=1}^{n} a_{j} e^{-\rho_{j}(x-u)}\left[1+\underline{\eta} B_{j}^{-1}\left(\exp \left\{u B_{j}\right\}-I_{m}\right) \underline{A}^{o}\right]$.

On the other hand, if $u>x$, then $I(u-z<x)=1$ for all $z \in(u-x, u]$, and so equation (3.4) becomes

$$
h(u, x, y)=\mathcal{C} \underline{\alpha} \exp \{(x+y) A\} \underline{A}^{o} \sum_{j=1}^{n} a_{j} e^{\rho_{j}(u-x)} \int_{u-x}^{u} e^{-\rho_{j} z} \underline{\eta} \exp \{z D\} \underline{A}^{o} d z .
$$

In a similar fashion which led to equation (3.9), we have

$$
\int_{u-x}^{u} e^{-\rho_{j} z} \underline{\eta} \exp \{z D\} \underline{A}^{o} d z=\underline{\eta} B_{j}^{-1}\left[\exp \left\{u B_{j}\right\}-\exp \left\{(u-x) B_{j}\right\}\right] \underline{A}^{o} .
$$

Therefore, for $u>x$, equation (3.11) becomes after some straightforward manipulation

$h(u, x, y)=\mathcal{C} \underline{\alpha} \exp \{(x+y) A\} \underline{A}^{o} \sum_{j=1}^{n} a_{j} \underline{\eta} B_{j}^{-1}\left[e^{-\rho_{j} x} \exp \{u D\}-\exp \{(u-x) D\}\right] \underline{A}^{o}$.

If we now integrate equations (3.10) and (3.12) from $y=0$ to $y=\infty$, we immediately obtain the following marginal density function of the surplus prior to ruin. For $0 \leq u<x$,

$$
f(u, x)=\mathcal{C} \underline{\alpha} \exp \{x A\} \underline{e}_{m} \sum_{j=1}^{n} a_{j} e^{-\rho_{j}(x-u)}\left[1+\underline{\eta} B_{j}^{-1}\left(\exp \left\{u B_{j}\right\}-I_{m}\right) \underline{A}^{o}\right],
$$

and for $u>x$

$$
f(u, x)=\mathcal{C} \underline{\alpha} \exp \{x A\} \underline{e}_{m} \sum_{j=1}^{n} a_{j} \underline{\eta} B_{j}^{-1}\left[e^{-\rho_{j} x} \exp \{u D\}-\exp \{(u-x) D\}\right] \underline{A}^{o} .
$$

The marginal density function of the deficit at ruin can also be obtained by integrating equation (3.12) from $x=0$ to $x=u$ followed by integrating equation (3.10) from $x=u$ to $x=\infty$. This is equivalent to integrating equation (3.4) from $x=0$ to $x=\infty$. After a fair bit of matrix algebra and simplification, it can be shown that

$$
g(u, y)=\underline{\eta} \exp \{u D\} \exp \{y A\} \underline{A}^{\circ}, y>0,
$$


a result which agrees with that of Drekic et al (2001, p.12).

We conclude this section with a few remarks. First of all, by comparing equations (3.13) and (3.14) as $x \rightarrow u^{-}$and $x \rightarrow u^{+}$, respectively, the density function $f(u, x)$ has a jump discontinuity at $x=u$ unless $\sum_{j=1}^{n} a_{j}=0$, in which case $f(u, x)$ is strictly continuous. Furthermore, in the classical model (i.e. when $k(t)=\lambda e^{-\lambda t}$ ), we have $n=1, \mathcal{C}=\lambda / c, \rho_{1}=0, a_{1}=1, B_{1}=D$, and $\underline{\eta}=-(\lambda / c) \underline{\alpha} A^{-1}$. With these values, it is a straightforward exercise to show that (i) equations (3.13) and (3.14) reduce to give equations (3.1) and (3.2), respectively, in Dickson (1992), and (ii) equation (3.15) agrees with the result given in Drekic et al (2001, p.13).

Finally we remark that the most obvious, and we believe useful, application of the generalised Erlang distribution is the Erlang distribution itself. Whilst there are many distributions in the class, the class is restrictive in the sense that the generalised Erlang density is a combination of densities and the parameters $\left\{\lambda_{j}\right\}_{j=1}^{n}$ determine the coefficients of the densities in the combination.

\section{Phase-type(2) inter-claim times}

In this section, we consider the case when $k$ is a phase-type(2) density function. In what follows, we adopt the notation and approach of Dickson and Hipp (2000) who observed that when $k$ is a phase-type(2) density function, it satisfies the differential equation

$$
k(t)+A_{1} k^{\prime}(t)+A_{2} k^{\prime \prime}(t)=0
$$

for $t>0$, where $A_{1}$ and $A_{2}$ are constants with $A_{2}>0$. Cheng and Tang (2003) considered the Erlang $(2, \sigma)$ case with $k(t)=\sigma^{2} t e^{-\sigma t}$, so that $A_{1}=2 / \sigma$ and $A_{2}=1 / \sigma^{2}$.

We begin by deriving an expression for $h(0, x, y)$ which is required to use equation (2.3). In order to do so, we use the function $\phi$ defined in equation (1.1) with $w\left(x_{1}, x_{2}\right)=I\left(x_{1} \leq x\right) I\left(x_{2} \leq y\right)$. However, we will initially work with a general function $w$. We start from equation (2.3) of Cheng and Tang (2003) which applies to any Sparre Andersen model:

$$
\begin{aligned}
c \phi(u)= & \int_{u}^{\infty} k\left(\frac{s-u}{c}\right) \int_{0}^{s} \phi(s-x) p(x) d x d s \\
& +\int_{u}^{\infty} k\left(\frac{s-u}{c}\right) \int_{s}^{\infty} w(s, x-s) p(x) d x d s \\
= & \int_{u}^{\infty} k\left(\frac{s-u}{c}\right)[\tau(s)+\omega(s)] d s
\end{aligned}
$$


where $\tau(u)=\int_{0}^{u} \phi(u-x) p(x) d x$ and $\omega(u)=\int_{u}^{\infty} w(u, x-u) p(x) d x$. Differentiation of equation (4.2) then yields

$$
c \phi^{\prime}(u)=-k(0)[\tau(u)+\omega(u)]-\frac{1}{c} \int_{u}^{\infty} k^{\prime}\left(\frac{s-u}{c}\right)[\tau(s)+\omega(s)] d s
$$

and hence

$$
\begin{aligned}
c \phi^{\prime \prime}(u)= & -k(0)\left[\tau^{\prime}(u)+\omega^{\prime}(u)\right]+\frac{1}{c} k^{\prime}(0)[\tau(u)+\omega(u)] \\
& +\frac{1}{c^{2}} \int_{u}^{\infty} k^{\prime \prime}\left(\frac{s-u}{c}\right)[\tau(s)+\omega(s)] d s .
\end{aligned}
$$

Following Dickson and Hipp (2000, p.152), we use equation (4.1) to obtain

$$
\begin{aligned}
& A_{2} c^{2} \phi^{\prime \prime}(u)-A_{1} c \phi^{\prime}(u)+\phi(u) \\
= & A_{2} k^{\prime}(0)[\tau(u)+\omega(u)]-A_{2} c k(0)\left[\tau^{\prime}(u)+\omega^{\prime}(u)\right]+k(0) A_{1}[\tau(u)+\omega(u)] \\
= & \tau(u)+\omega(u)-A_{2} c k(0)\left[\tau^{\prime}(u)+\omega^{\prime}(u)\right]
\end{aligned}
$$

since $A_{1} k(0)+A_{2} k^{\prime}(0)=1$ (see Dickson and Hipp (2000, equation (2.1))). Taking the Laplace transform of this equation, and noting that $\tau$ is a convolution, we obtain

$$
\begin{aligned}
& A_{2} c^{2}\left[s^{2} \phi^{*}(s)-s \phi(0)-\phi^{\prime}(0)\right]-A_{1} c\left[s \phi^{*}(s)-\phi(0)\right]+\phi^{*}(s) \\
= & \phi^{*}(s) p^{*}(s)+\omega^{*}(s)-A_{2} c k(0)\left[s \phi^{*}(s) p^{*}(s)+s \omega^{*}(s)-\omega(0)\right]
\end{aligned}
$$

so that

$$
\phi^{*}(s)=\frac{A_{2} c^{2}\left[s \phi(0)+\phi^{\prime}(0)\right]-A_{1} c \phi(0)+\omega^{*}(s)-A_{2} c k(0)\left[s \omega^{*}(s)-\omega(0)\right]}{A_{2} c^{2} s^{2}-A_{1} c s+1-p^{*}(s)+A_{2} c k(0) s p^{*}(s)},
$$

or, equivalently

$$
s \phi^{*}(s)=\frac{A_{2} c^{2}\left[s \phi(0)+\phi^{\prime}(0)\right]-A_{1} c \phi(0)+\omega^{*}(s)-A_{2} c k(0)\left[s \omega^{*}(s)-\omega(0)\right]}{A_{2} c^{2} s-A_{1} c+\kappa q^{*}(s)+A_{2} c k(0) p^{*}(s)}
$$

where we define $q^{*}(s)=\left[1-p^{*}(s)\right] /(\kappa s)$. Dickson and Hipp (2000, Section 3 ) show that there is a unique positive number $s_{0}$ such that

$$
A_{2} c^{2} s_{0}-A_{1} c+\kappa q^{*}\left(s_{0}\right)+A_{2} c k(0) p^{*}\left(s_{0}\right)=0 .
$$

As $s_{0}$ is a zero of the denominator in equation (4.3), it must also be a zero of the numerator. Making use of this observation, we write

$$
s \phi^{*}(s)=\frac{A_{2} c^{2}\left(s-s_{0}\right) \phi(0)+\omega^{*}(s)-\omega^{*}\left(s_{0}\right)-A_{2} c k(0)\left[s \omega^{*}(s)-s_{0} \omega^{*}\left(s_{0}\right)\right]}{A_{2} c^{2} s-A_{1} c+\kappa q^{*}(s)+A_{2} c k(0) p^{*}(s)} .
$$


If we now set $s=0$ in the denominator, we get $-A_{1} c+\kappa+A_{2} c k(0)=\kappa-c \mu \neq$ 0 (see Dickson and Hipp (2000, equation (2.3))). Hence the numerator must be zero with $s=0$ as $\lim _{s \rightarrow 0^{+}} s \phi^{*}(s)=0$. Thus

$$
-A_{2} c^{2} s_{0} \phi(0)+\omega^{*}(0)-\omega^{*}\left(s_{0}\right)+A_{2} c k(0) s_{0} \omega^{*}\left(s_{0}\right)=0
$$

giving

$$
\phi(0)=\frac{1}{A_{2} c^{2} s_{0}}\left[\omega^{*}(0)-\omega^{*}\left(s_{0}\right)\right]+\frac{k(0)}{c} \omega^{*}\left(s_{0}\right)
$$

We remark that equation (4.4) generalises Corollary 2.3 of Cheng and Tang (2003) as their $r_{2}$ is our $s_{0}$.

Now let $w\left(x_{1}, x_{2}\right)=I\left(x_{1} \leq x\right) I\left(x_{2} \leq y\right)$, so that $\phi(0)=H(0, x, y)$. It then follows that

$$
\begin{aligned}
\omega(u) & =\int_{u}^{\infty} I(u \leq x) I(r-u \leq y) p(r) d r \\
& =I(u \leq x) \int_{u}^{\infty} I(r \leq u+y) p(r) d r \\
& =I(u \leq x)[\bar{P}(u)-\bar{P}(u+y)] .
\end{aligned}
$$

This gives

$$
\omega^{*}(0)=\int_{0}^{\infty} I(u \leq x)[\bar{P}(u)-\bar{P}(u+y)] d u=\int_{0}^{x}[\vec{P}(u)-\bar{P}(u+y)] d u
$$

and

$$
\omega^{*}\left(s_{0}\right)=\int_{0}^{x} e^{-s_{0} u}[\bar{P}(u)-\bar{P}(u+y)] d u,
$$

thereby yielding

$$
\begin{aligned}
H(0, x, y)= & \frac{1}{A_{2} c^{2} s_{0}} \int_{0}^{x}[\bar{P}(u)-\bar{P}(u+y)] d u \\
& -\frac{1-A_{2} c k(0) s_{0}}{A_{2} c^{2} s_{0}} \int_{0}^{x} e^{-s_{0} u}[\bar{P}(u)-\bar{P}(u+y)] d u .
\end{aligned}
$$

Differentiating equation (4.5) with respect to $x$ and then $y$ gives

$$
\begin{aligned}
h(0, x, y) & =\frac{1}{A_{2} c^{2} s_{0}}\left\{p(x+y)-\left[1-A_{2} c k(0) s_{0}\right] e^{-s_{0} x} p(x+y)\right\} \\
& =\frac{1}{A_{2} c^{2} s_{0}}\left(1-e^{-s_{0} x}\right) p(x+y)+\frac{k(0)}{c} e^{-s_{0} x} p(x+y)
\end{aligned}
$$

We can easily verify that

$$
h(0, x, y)=f(0, x) \frac{p(x+y)}{\bar{P}(x)}
$$


by first letting $y \rightarrow \infty$ in equation (4.5) and then differentiating the result with respect to $x$.

We remark that equation (4.6) generalises equation (3.9) of Cheng and Tang (2003) with $u=0$, as their $k(0)$ is equal to 0 . Also, note that equation (4.6) may be expressed in the form of equation (3.2) by setting $n=2, \mathcal{C}=1$, $\rho_{1}=0, \rho_{2}=s_{0}, a_{1}=\left(A_{2} c^{2} s_{0}\right)^{-1}$, and $a_{2}=k(0) / c-\left(A_{2} c^{2} s_{0}\right)^{-1}$. Moreover, it is readily verified that $\xi=0$ and $\xi=s_{0}$ are the two distinct roots in the right half of the complex plane satisfying $k^{*}(-c \xi) p^{*}(\xi)=1$. Therefore, if claim amounts have a phase-type $(m)$ distribution, then equations (3.4) through to (3.15) continue to hold (with constants assigned as above) in the case when inter-claim times are phase-type(2) distributed.

\section{Examples}

In this section, we present three examples to illustrate the results of the previous sections. The results in Examples 5.2 and 5.3 were obtained with the aid of the symbolic computational package Mathematica.

Example 5.1 We start with a straightforward example for which the phasetype machinery is not required. Let $p(x)=\nu e^{-\nu x}, x>0$, and let $k(t)=$ $4 t e^{-2 t}, t>0$ (i.e. exponential claim amounts and Erlang(2,2) inter-claim times). In this case, $k(0)=0$ and $A_{2}=1 / 4$, so that equation (4.6) becomes

$$
h(0, x, y)=\frac{4 \nu}{c^{2} s_{0}}\left(1-e^{-s_{0} x}\right) e^{-\nu(x+y)} .
$$

It is well known (see, for example, Rolski et al (1999, Section 6.5.1)) that

$$
\delta(u)=1-(1-R / \nu) e^{-R u}
$$

where $R$ is the adjustment coefficient. Hence, for $0 \leq u<x$,

$$
\begin{aligned}
h(u, x, y) & =\frac{1}{\delta(0)} \int_{0}^{u} h(0, x-u+z, u-z+y) d \delta(z) \\
= & \frac{4 \nu e^{-\nu(x+y)}}{c^{2} s_{0}}\left[\left(1-e^{-s_{0}(x-u)}\right)+\frac{\nu-R}{R}\left(1-e^{-R u}\right)\right. \\
& \left.\quad-\frac{\nu-R}{s_{0}+R} e^{-s_{0}(x-u)}\left(1-e^{-\left(s_{0}+R\right) u}\right)\right]
\end{aligned}
$$

and integrating out $y$ we obtain

$$
\begin{aligned}
f(u, x)=\frac{4 e^{-\nu x}}{c^{2} s_{0}}\left[\left(1-e^{-s_{0}(x-u)}\right)\right. & +\frac{\nu-R}{R}\left(1-e^{-R u}\right) \\
& \left.-\frac{\nu-R}{s_{0}+R} e^{-s_{0}(x-u)}\left(1-e^{-\left(s_{0}+R\right) u}\right)\right] .
\end{aligned}
$$


Similarly, for $u>x$,

$$
\begin{aligned}
h(u, x, y) & =\frac{1}{\delta(0)} \int_{u-x}^{u} h(0, x-u+z, u-z+y) d \delta(z) \\
& =(\nu-R) \frac{4 \nu e^{-\nu(x+y)}}{c^{2} s_{0}} e^{-R u}\left[\frac{1}{R}\left(e^{R x}-1\right)-\frac{e^{R x}-e^{-s_{0} x}}{s_{0}+R}\right]
\end{aligned}
$$

and

$$
f(u, x)=(\nu-R) \frac{4 e^{-\nu x}}{c^{2} s_{0}} e^{-R u}\left[\frac{1}{R}\left(e^{R x}-1\right)-\frac{e^{R x}-e^{-s_{0} x}}{s_{0}+R}\right] .
$$

We remark that these results can also be obtained from Example 1 of Li and Garrido (2003) by setting $n=2$ there.

Example 5.2 Consider an inter-claim time distribution that is an equal mixture of two exponentials at rates $1 / 3$ and 1 respectively, so that

$$
k(t)=\frac{1}{2} e^{-t}+\frac{1}{6} e^{-t / 3}, \quad t>0 .
$$

We remark that this distribution belongs to the phase-type(2) class but is not a member of the generalised Erlang family. Moreover, we have $\mu=2$, $k(0)=2 / 3, A_{1}=4$, and $A_{2}=3$. Suppose that the individual claim amount distribution is Erlang(3,1.5), so that

$$
p(x)=\frac{27}{16} x^{2} e^{-1.5 x}, \quad x>0, \quad \text { and } \bar{P}(x)=e^{-1.5 x}\left(1+\frac{3}{2} x+\frac{9}{8} x^{2}\right), \quad x \geq 0 .
$$

We note that $\kappa=2$. Written in phase-type form, we have $\underline{\alpha}=(1,0,0)$, $\underline{A}^{o}=(0,0,1.5)^{\prime}$, and

$$
A=\left[\begin{array}{ccc}
-1.5 & 1.5 & 0 \\
0 & -1.5 & 1.5 \\
0 & 0 & -1.5
\end{array}\right]
$$

Let $\theta=0.1$ so that $c=1.1$. Then we obtain (to 5 decimal places of accuracy) $s_{0}=0.79184, \underline{\eta}=(0.34458,0.30566,0.28019)$, and

$$
D=\left[\begin{array}{ccc}
-1.50000 & 1.50000 & 0 \\
0 & -1.50000 & 1.50000 \\
0.51687 & 0.45849 & -1.07972
\end{array}\right]
$$

As $D$ has one real eigenvalue $(-0.05110)$ and two complex ones $(-2.0143 \pm$ $0.73357 i)$, expressions for $h(u, x, y)$ will involve trigonometric functions. For 
$0 \leq u<x$, we obtain

$$
\begin{aligned}
h(u, x, y)= & p(x+y)\left\{5-\left(4.7147+0.21209 e^{-0.79184 x}\right) e^{-0.0511 u}\right. \\
& +0.43824 e^{-0.79184(x-u)} \\
& +\left(0.06263+0.032 e^{-0.79184 x}\right) e^{-2.0143 u} \operatorname{Cos}[0.73357 u] \\
& \left.+\left(0.04288+0.02663 e^{-0.79184 x}\right) e^{-2.0143 u} \operatorname{Sin}[0.73357 u]\right\}
\end{aligned}
$$

and for $u>x$

$$
\begin{aligned}
h(u, x, y)= & p(x+y)\left\{-\left(4.7147+0.21209 e^{-0.79184 x}\right) e^{-0.0511 u}\right. \\
& +4.92682 e^{-0.0511(u-x)} \\
& +\left(0.06263+0.032 e^{-0.79184 x}\right) e^{-2.0143 u} \operatorname{Cos}[0.73357 u] \\
& +\left(0.04288+0.02663 e^{-0.79184 x}\right) e^{-2.0143 u} \operatorname{Sin}[0.73357 u] \\
& -0.09464 e^{-2.0143(u-x)} \operatorname{Cos}[0.73357(u-x)] \\
& \left.-0.06951 e^{-2.0143(u-x)} \operatorname{Sin}[0.73357(u-x)]\right\} .
\end{aligned}
$$

The density function $f(u, x)$ has the same form as the two equations above, except that $p(x+y)$ is replaced by $\bar{P}(x)$. Figures 1 and 2 display the shape of $f(u, x)$ for $u=1$ and $u=3$, respectively. The density functions are very similar to those illustrated by Dufresne and Gerber (1988) and Dickson (1992). Since $k(0)=2 / 3 \neq 0$, we have $a_{1}+a_{2} \neq 0$. Based on our earlier remark at the end of Section 3, this implies that $f(u, x)$ possesses a jump discontinuity at $x=u$. This is indeed the case as evidenced by Figures 1 and 2, although it becomes more difficult to tell for larger values of $u$. Finally, the density function $g(u, y)$ is

$$
\begin{aligned}
g(u, y)= & \left(0.6925+0.71232 y+0.27792 y^{2}\right) e^{-0.0511 u-1.5 y} \\
& -\left(0.27222+0.02459 y-0.30356 y^{2}\right) e^{-2.0143 u-1.5 y} \operatorname{Cos}[0.73357 u] \\
& -\left(0.38035-0.55516 y+0.00296 y^{2}\right) e^{-2.0143 u-1.5 y} \operatorname{Sin}[0.73357 u] .
\end{aligned}
$$

Example 5.3 Suppose inter-claim times have a generalised Erlang distribution with $n=3, \lambda_{1}=\lambda_{2}=0.5$, and $\lambda_{3}=2$. With these values, equation (3.1) simplifies to give

$$
k(t)=\frac{2}{9} e^{-2 t}-\frac{2}{9} e^{-0.5 t}+\frac{1}{3} t e^{-0.5 t}, \quad t>0
$$

with $\mu=4.5$. Let the individual claim amounts be distributed with phase-type representation $\underline{\alpha}=(0.1,0.1,0.3,0.5), \underline{A}^{o}=(0,0,0,0.5)^{\prime}$, and

$$
A=\left[\begin{array}{cccc}
-1 / 10 & 1 / 10 & 0 & 0 \\
0 & -1 / 6 & 1 / 6 & 0 \\
0 & 0 & -1 / 3 & 1 / 3 \\
0 & 0 & 0 & -1 / 2
\end{array}\right]
$$


Then the mean claim amount is $\kappa=5.7$. Moreover, we have

$$
p(x)=\frac{5}{112} e^{-x / 10}-\frac{1}{40} e^{-x / 6}+\frac{17}{70} e^{-x / 3}-\frac{1}{80} e^{-x / 2}, \quad x>0,
$$

and

$$
\bar{P}(x)=\frac{25}{56} e^{-x / 10}-\frac{3}{20} e^{-x / 6}+\frac{51}{70} e^{-x / 3}-\frac{1}{40} e^{-x / 2}, \quad x \geq 0 .
$$

Let $\theta=0.2$ so that $c=1.52$. Then we obtain (to 5 decimal places of accuracy) $\mathcal{C}=0.14238, \rho_{1}=0, \rho_{2}=0.56407, \rho_{3}=1.29160$,

$$
\underline{\eta}=(0.15407,0.19102,0.19612,0.23601),
$$

and

$$
D=\left[\begin{array}{cccc}
-0.10000 & 0.10000 & 0 & 0 \\
0 & -0.16667 & 0.16667 & 0 \\
0 & 0 & -0.33333 & 0.33333 \\
0.07703 & 0.09551 & 0.09806 & -0.38199
\end{array}\right]
$$

We note that $D$ has real eigenvalues, namely $-0.50122,-0.27485,-0.18112$, and -0.02480 . Therefore, for $0 \leq u<x$, we obtain

$$
\begin{aligned}
h(u, x, y)= & 0.14238 p(x+y)\left[6.1611+0.00217 e^{-0.50122 u}-0.23444 e^{-0.27485 u}\right. \\
& +0.09197 e^{-0.18112 u}-4.6482 e^{-0.0248 u}+1.1547 e^{-1.2916(x-u)}-2.8792 e^{-0.56407(x-u)} \\
& +\left(0.00047 e^{-0.50122 u}-0.03189 e^{-0.27485 u}+0.00877 e^{-0.18112 u}\right. \\
& \left.-0.0679 e^{-0.0248 u}\right) e^{-1.2916 x}-\left(0.00182 e^{-0.50122 u}-0.13636 e^{-0.27485 u}\right. \\
& \left.\left.+0.03969 e^{-0.1812 u}-0.34755 e^{-0.0248 u}\right) e^{-0.56407 x}\right]
\end{aligned}
$$

and for $u>x$

$$
\begin{aligned}
h(u, x, y)= & 0.14238 p(x+y)\left[0.00217 e^{-0.50122 u}-0.23444 e^{-0.27485 u}+0.09197 e^{-0.18112 u}\right. \\
& -4.6482 e^{-0.0248 u}+4.3685 e^{-0.0248(u-x)}-0.06106 e^{-0.18112(u-x)} \\
& +0.12998 e^{-0.27485(u-x)}-0.00083 e^{-0.50122(u-x)}+\left(0.00047 e^{-0.50122 u}\right. \\
& \left.-0.03189 e^{-0.27485 u}+0.00877 e^{-0.18112 u}-0.0679 e^{-0.0248 u}\right) e^{-1.2916 x} \\
& -\left(0.00182 e^{-0.50122 u}-0.13636 e^{-0.27485 u}+0.03969 e^{-0.18112 u}\right. \\
& \left.\left.-0.34755 e^{-0.0248 u}\right) e^{-0.56407 x}\right] .
\end{aligned}
$$

As we commented in Example 5.2, the density function $f(u, x)$ has the same form as the two equations above, except that $p(x+y)$ is replaced by $\bar{P}(x)$. Figures 3 and 4 display the shape of $f(u, x)$ for $u=20$ and $u=40$, respectively. Since $a_{1}+a_{2}+a_{3}=1.37257-2.43676+1.06419=0, f(u, x)$ is 
strictly continuous for all $x>0$, as evidenced by Figures 3 and 4. For the sake of completeness, the density function $g(u, y)$ is given by

$$
\begin{aligned}
& g(u, y)= \\
& \left(0.00014 e^{-0.50122 u}-0.01847 e^{-0.27485 u}+0.01029 e^{-0.18112 u}+0.07682 e^{-0.0248 u}\right) e^{-y / 10} \\
& -\left(0.00005 e^{-0.50122 u}-0.0087 e^{-0.27485 u}+0.01683 e^{-0.18112 u}+0.01187 e^{-0.0248 u}\right) e^{-y / 6} \\
& +\left(0.00034 e^{-0.50122 u}+0.05711 e^{-0.27485 u}-0.00567 e^{-0.18112 u}+0.01936 e^{-0.0248 u}\right) e^{-y / 3} \\
& -\left(0.00122 e^{-0.50122 u}+0.00039 e^{-0.27485 u}-0.00007 e^{-0.18112 u}+0.00033 e^{-0.0248 u}\right) e^{-y / 2} .
\end{aligned}
$$

\section{References}

[1] Asmussen, S. (2000) Ruin Probabilities. World Scientific Publishing, Singapore.

[2] Cheng, Y. and Tang, Q. (2003) Moments of the surplus before ruin and the deficit at ruin in the Erlang(2) risk process. North American Actuarial Journal 7, 1-12.

[3] Dickson, D. C. M. (1992) On the distribution of the surplus prior to ruin. Insurance: Mathematics \& Economics 11, 191-207.

[4] Dickson, D. C. M. and Hipp, C. (2000) Ruin problems for phase-type(2) risk processes. Scandinavian Actuarial Journal, 147-167.

[5] Drekic, S., Dickson, D. C. M., Stanford, D. A., and Willmot, G. E. (2001) On the distribution of the deficit at ruin when claims are phasetype. IIPR Research Report 01-12, Department of Statistics and Actuarial Science, University of Waterloo. To appear in the Scandinavian Actuarial Journal.

[6] Dufresne, F. and Gerber, H. U. (1988) The surpluses immediately before and at ruin, and the amount of the claim causing ruin. Insurance: Mathematics \& Economics 7, 193-199.

[7] Gerber, H. U. and Shiu, E. S. W. (1998) On the time value of ruin. North American Actuarial Journal 2, 48-78.

[8] Gerber, H. U. and Shiu, E. S. W. (2003a) First discussion of Y. Cheng and Q. Tang's "Moments of the surplus before ruin and the deficit at ruin in the Erlang(2) risk process". To appear in the North American Actuarial Journal. 
[9] Gerber, H. U. and Shiu, E. S. W. (2003b) Second discussion of Y. Cheng and Q. Tang's "Moments of the surplus before ruin and the deficit at ruin in the Erlang(2) risk process". To appear in the North American Actuarial Journal.

[10] Latouche, G. and Ramaswami, V. (1999) Introduction to Matrix Analytic Methods in Stochastic Modeling. ASA SIAM, Philadelphia.

[11] Li, S. and Garrido, J. (2003) On ruin for the Erlang $(n)$ risk process. Technical Report No. 1/03, Department of Mathematics and Statistics, Concordia University.

[12] Neuts, M. F. (1981) Matrix-geometric Solutions in Stochastic Models: An Algorithmic Approach. John Hopkins University Press, Baltimore.

[13] Rolski, T., Schmidli, H., Schmidt, V., and Teugels, J. (1999) Stochastic Processes for Insurance and Finance. John Wiley, Chichester.

[14] Willmot, G. E. and Lin, X. (1998) Exact and approximate properties of the distribution of the surplus before ruin. Insurance: Mathematics $\&$ Economics 23, 91-110.

\author{
David C M Dickson \\ Centre for Actuarial Studies \\ Department of Economics \\ University of Melbourne \\ Victoria 3010 \\ Australia \\ email: dcmd@unimelb.edu.au
}

\author{
Steve Drekic \\ Department of Statistics and \\ Actuarial Science \\ University of Waterloo \\ Waterloo, Ontario \\ Canada N2L 3G1 \\ email: sdrekic@math.uwaterloo.ca
}




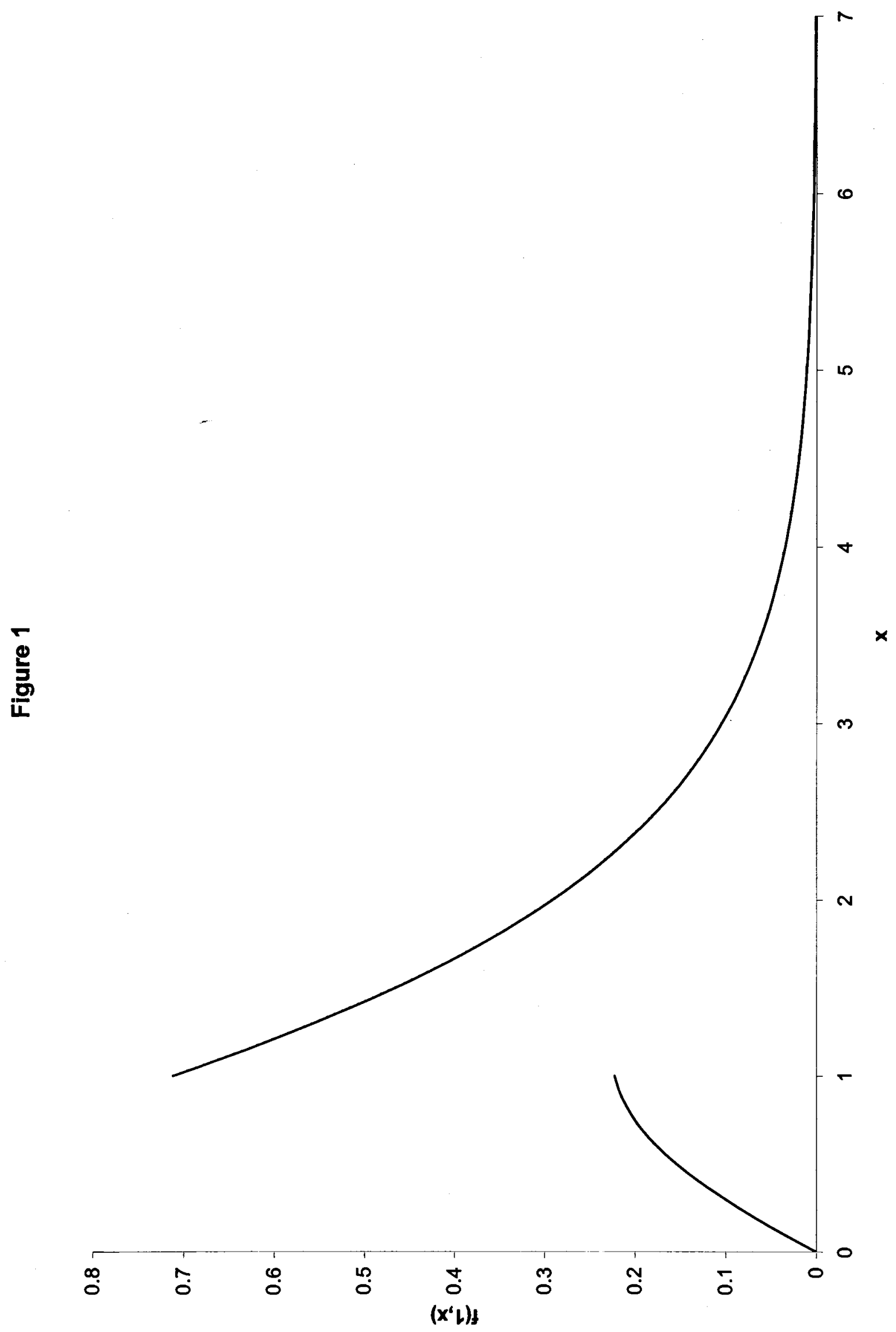




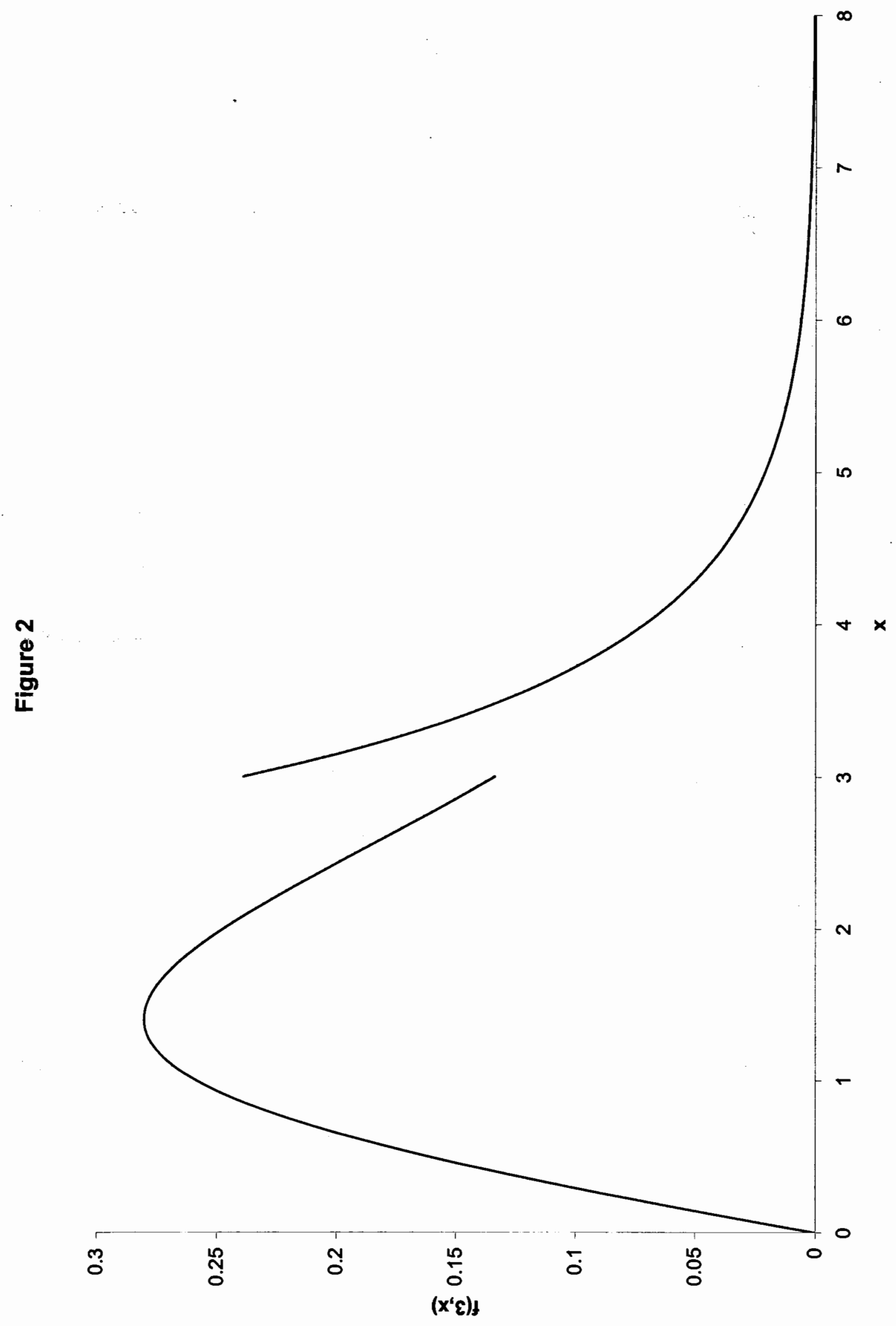




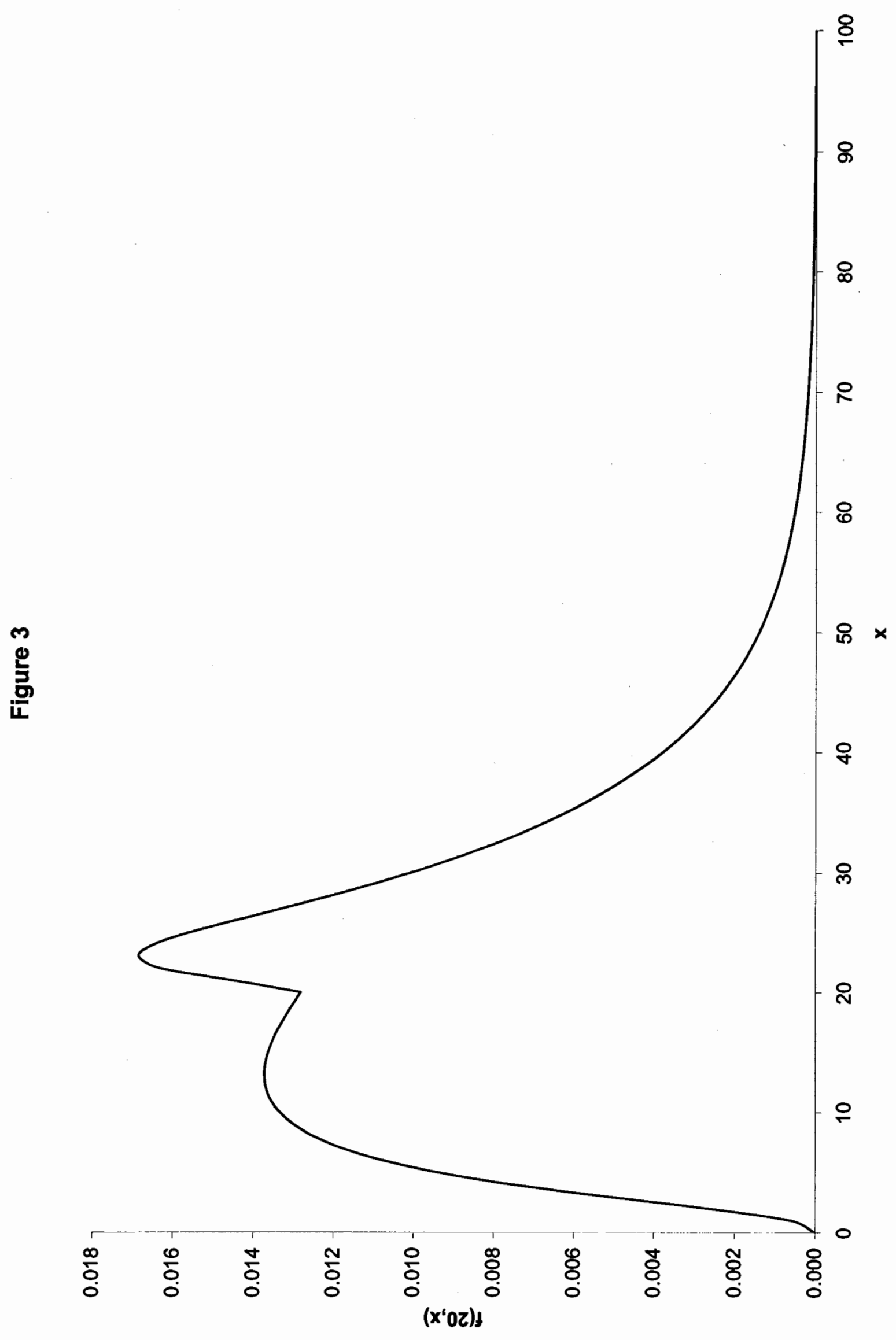




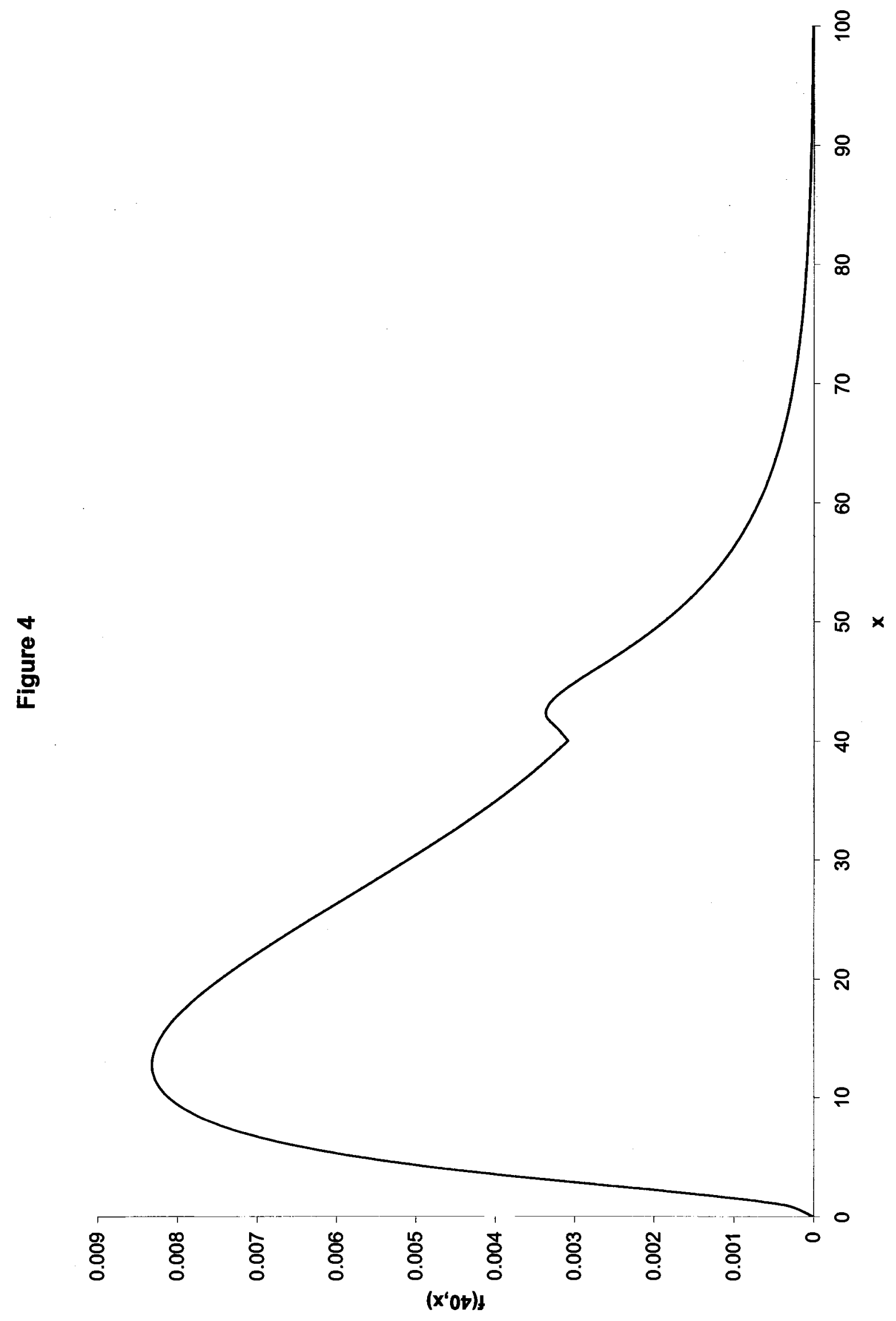


No.

Date

FEBRUARY 2001

FEBRUARY 2001

JUNE 2001

SEPTEMBER 2001

NOVEMBER 2001

NOVEMBER 2001

NOVEMBER 2001

JANUARY 2002

JANUARY 2002

JANUARY 2002

JANUARY 2002

MAY 2002

JUNE 2002

JUNE 2002
NOVEMBER 2001

Subject

Author

DISCRETE TIME RISK MODELS UNDER STOCHASTIC FORCES OF INTEREST

MODERN LANDMARKS IN ACTUARIAL SCIENCE Inaugural Professorial Address

LUNDBERG INEQUALITIES FOR RENEWAL EQUATIONS

VOLATILITY, BETA AND RETURN WAS THERE EVER A MEANINGFUL RELATIONSHIP?

EXPLICIT, FINITE TIME RUIN PROBABILITIES FOR DISCRETE, DEPENDENT CLAIMS

ON THE DISTRIBUTION OF THE DEFICIT AT RUIN WHEN CLAIMS ARE PHASE-TYPE

THE INTEGRATED SQUARE-ROOT PROCESS

ON THE EXPECTED DISCOUNTED PENALTY FUNCTION AT RUIN OF A SURPLUS PROCESS WITH INTEREST

\section{CHAIN LADDER BIAS}

FURTHER OBSERVATIONS ON CHAIN LADDER BIAS

A GENERAL CLASS OF RISK MODELS

THE DISTRIBUTION OF THE TIME TO RUIN IN THE CLASSICAL RISK MODEL

A NOTE ON THE MAXIMUM SEVERITY OF RUIN AND RELATED PROBLEMS

UPPER BOUNDS FOR ULTIMATE RUIN PROBABILITIES IN THE SPARRE ANDERSEN MODEL WITH INTEREST
Jun Cai

David C M Dickson

Gordon E Willmot

Jun Cai

$\mathrm{X}$ Sheldon Lin

Richard Fitzherbert

Zvetan G Ignatov Vladimir K Kaishev Rossen S Krachunov

Steve Drekic

David C M Dickson

David A Stanford

Gordon E Willmot

Daniel Dufresne

Jun Cai

David C M Dickson

Greg Taylor

Greg Taylor

Daniel Dufresne

David C M Dickson Howard R Waters

David C M Dickson Jun Cai

David C M Dickson

Richard Fitzherbert

\author{
CONTINUOUS COMPOUNDING, VOLATILITY AND \\ THE EQUITY PREMIUM
}




\begin{tabular}{|c|c|c|c|}
\hline No. & Date & Subject & Author \\
\hline 99 & JUNE 2002 & $\begin{array}{l}\text { THE DEFICIT AT RUIN IN THE STATIONARY } \\
\text { RENEWAL RISK MODEL }\end{array}$ & $\begin{array}{l}\text { Gordon E Willmot } \\
\text { David C M Dickson } \\
\text { Steve Drekic } \\
\text { David A Stanford }\end{array}$ \\
\hline 100 & AUGUST 2002 & ASIAN AND BASKET ASYMPTOTICS & Daniel Dufresne \\
\hline 101 & AUGUST 2002 & $\begin{array}{l}\text { RUIN PROBABILITIES WITH A MARKOV CHAIN } \\
\text { INTEREST MODEL }\end{array}$ & $\begin{array}{l}\text { Jun Cai } \\
\text { David C M Dickson }\end{array}$ \\
\hline 102 & AUGUST 2002 & $\begin{array}{l}\text { THE GERBER-SHIU DISCOUNTED PENALTY } \\
\text { FUNCTION IN THE STATIONARY RENEWAL RISK } \\
\text { MODEL }\end{array}$ & $\begin{array}{l}\text { Gordon E Willmot } \\
\text { David C M Dickson }\end{array}$ \\
\hline 103 & NOVEMBER 2002 & $\begin{array}{l}\text { INITIAL CAPITAL AND MARGINS REQUIRED TO } \\
\text { SECURE A JAPANESE LIFE INSURANCE POLICY } \\
\text { PORTFOLIO UNDER VARIABLE INTEREST RATES }\end{array}$ & $\begin{array}{l}\text { Manabu Sato } \\
\text { David C M Dickson } \\
\text { Richard M Fitzherbert }\end{array}$ \\
\hline 104 & NOVEMBER 2002 & STATISTICAL CASE ESTIMATION & $\begin{array}{l}\text { Greg Taylor } \\
\text { Mireille Campbell }\end{array}$ \\
\hline 105 & MARCH 2003 & $\begin{array}{l}\text { ACTUARIAL PRACTICE AND CONTROL: } \\
\text { OBJECTIVES AND CAPABILITIES }\end{array}$ & Julian D Gribble \\
\hline 106 & MARCH 2003 & $\begin{array}{l}\text { THE IDENTIFICATION AND MEASUREMENT OF } \\
\text { SPECULATIVE RISK }\end{array}$ & Richard Fitzherbert \\
\hline 107 & AUGUST 2003 & SOME OPTIMAL DIVIDENDS PROBLEMS & $\begin{array}{l}\text { David C M Dickson } \\
\text { Howard R Waters }\end{array}$ \\
\hline 108 & AUGUST 2003 & $\begin{array}{l}\text { THE JOINT DISTRIBUTION OF THE SURPLUS } \\
\text { PRIOR TO RUIN AND THE DEFICIT AT RUIN IN } \\
\text { SOME SPARRE ANDERSEN MODELS }\end{array}$ & $\begin{array}{l}\text { David C M Dickson } \\
\text { Steve Drekic }\end{array}$ \\
\hline
\end{tabular}

A complete list of papers can be found at http://www.economics.unimelb.edu.au/actwww/papers.html 


\section{University Library}

\section{- M M I N E R VA A gateway to Melbourne's research publications}

Minerva Access is the Institutional Repository of The University of Melbourne

Author/s:

DICKSON, DCM;DREKIC, S

Title:

The joint distribution of the surplus prior to ruin and the deficit at ruin in some Sparre Andersen models

Date:

2004

Citation:

DICKSON, D. C. M. \& DREKIC, S. (2004). The joint distribution of the surplus prior to ruin and the deficit at ruin in some Sparre Andersen models. Insurance: Mathematics \& Economics, 34 (1), pp.97-107. https://doi.org/10.1016/j.insmatheco.2003.11.003.

Persistent Link:

http://hdl.handle.net/11343/33767 